\title{
Stability analysis of magnetic fluids in the presence of an oblique field and mass and heat transfer
}

\author{
Rabah Djeghiour ${ }^{1, *}$, Bachir Meziani ${ }^{1}$ \\ ${ }^{1}$ Laboratoire de Physique Théorique, Département de Physique, Université de Bejaia, 06000 Bejaia, Algeria
}

\begin{abstract}
In this paper, we investigate an analysis of the stability of a basic flow of streaming magnetic fluids in the presence of an oblique magnetic field is made. We have use the linear analysis of modified Kelvin-Helmholtz instability by the addition of the influence of mass transfer and heat across the interface. Problems equations model is presented where nonlinear terms are neglected in model equations as well as the boundary conditions. In the case of a oblique magnetic field, the dispersion relation is obtained and discussed both analytically and numerically and the stability diagrams are also obtained. It is found that the effect of the field depends strongly on the choice of some physical parameters of the system. Regions of stability and instability are identified. It is found that the mass and heat transfer parameter has a destabilizing influence regardless of the mechanism of the field.
\end{abstract}

\section{Introduction}

Kelvin-Helmholtz instability is one of the basic instabilities of two-fluid systems, which affects an interface. In this case is that with one layer of lighter fluid overlying another of denser fluid, and the two moving horizontally in the same direction but with different velocities.

When two fluids are divided by an interface, the interfacial instability is usually discussed without considering heat and mass transfer across the interface. On the other hand, the transfer of mass and heat across the interface is of great importance in numerous industrial and environmental processes. These include the design of many types of contacting equipment, e.g. boilers, condensers, evaporators, gas absorbers, piplines, chemical reactors, nuclear reactors, and in other problems such as the aeration of rivers and the sea.

Hsieh [1] formulated the general problem of interfacial fluid flow with mass and heat transfer and applied it to discuss the Kelvin-Helmholtz instability problem. Lee [2] has studied the Kelvin- Helmholtz instability of inviscid fluids taking heat and mass transfer into the account and observed that the heat and mass transfer has no effect on the linear inviscid analysis while it plays an important role in the nonlinear analysis. Heat and mass transfer effects on the Kelvin-Helmholtz instability of miscible and viscous fluids using viscous potential flow theory was considered by Asthana and Agrawal [3]. They observed that heat and mass transfer have a strong stabilizing effect when the lower fluid is highly viscous and a weak destabilizing effect when the fluid's viscosity is low.
The effect of a magnetic field on the stability of cylindrical flow with mass and heat transfer has been investigated by Elhefnawy and Radwan [4]. They found that the instability criteria is independent of mass and heat transfer coefficients, but it is different from that in the same problem without heat and mass transfer. On the other hand, Elhefnawy[5] studied the nonlinear analysis of the Rayleigh-Taylor stability of two superposed magnetic fluids with interfacial transfer of mass and heat is presented for two layers each of finite thickness in the presence of a uniform tangential magnetic field. It is found that, in the linear theory, the stability criterion is independent of mass and heat transfer coefficient. While in the nonlinear theory it is found that, when this coefficient is large enough, the system which would be unstable classically, can be stabilized for finite amplitude disturbances.

The objective of the present work is to study the effect of the oblique magnetic field on the KelvinHelmholtz instability of the interface separating two superposed magnetic fluids, with finite depth, when there is heat and mass transfer across the interface. We first present the mathematical formulation of the problem with the boundary conditions by adopting the linear theory. We will solve these equations to determine the system dispersion relation. Stability and instability are discussed both theoretically and numerically in the absence and presence of mass and heat transfer. The effect of nonlinearity on the problem at hand will not be discussed here.

\section{Formulation of the problem}

We consider a system of two incompressible and inviscid magnetic fluids, separated by a plane

\footnotetext{
* Corresponding author: rabah.djeghiour@gmail.com
} 
interface $y=0$. The lower fluid (1) occupies the region $-h_{1}<y<0$, having density $\rho_{1}$, magnetic permeability $\mu_{1}$, velocity $v_{1}$ and is bounded from below by the rigid plane surface $y=-h_{1}$. The upper fluid (2) occupies the region $0<y<h_{2}$, having density $\rho_{2}$, magnetic permeability $\mu_{2}$, velocity $v_{2}$ and is bounded from upper by the rigid plane surface $y=h_{2}$. The temperatures at $y=-h_{1}, y=0$ and $y=h_{2}$ are $T_{1}, T_{0}$ and $T_{2}$, respectively, where $T_{1}<T_{0}<T_{2}$ and surface tension at the interface is taken as $\sigma$.

The two fluids are influenced by an oblique magnetic field:

$$
H^{(i)}=H_{01}^{(i)} \vec{e}_{x}+H_{02}^{(i)} \vec{e}_{y} \quad i=1,2
$$

where $\vec{e}_{x}$ and $\vec{e}_{y}$ are unit vectors in the $x$ and $y$ directions, respectively.

We assume that there are no free currents at the separation surface at the equilibrium state. Therefore, magnetic field tangential components are continuous at the interface, while normal ones are discontinuous, i.e.

$$
\begin{gathered}
H_{01}^{(1)}=H_{01}^{(2)} \\
\mu^{(1)} H_{02}^{(1)}=\mu^{(2)} H_{02}^{(2)}
\end{gathered}
$$

In magneto-quasi-static case, we have a system with negligible displacement current. Maxwell's equations in absence of free currents are:

$$
\begin{array}{ll}
\nabla\left(\mu^{(i)} H^{(i)}\right)=0 & i=1,2 \\
\nabla \times H^{(i)}=0 & i=1,2
\end{array}
$$

In accordance with the validity of quasi-static approximation, a potential function $\Psi(x, y, t)$ may be introduced such that:

$$
H^{(i)}=-\nabla \Psi^{(i)} \quad i=1,2
$$

The total oblique magnetic field is given by:

$$
\vec{H}^{(i)}=\left(H_{01}^{(i)}-\Psi_{x}^{(i)}\right) \vec{e}_{x}+\left(H_{02}^{(i)}-\Psi_{y}^{(i)}\right) \vec{e}_{y} \quad i=1,2
$$

\subsection{Mathematical formulation of the problem}

Assuming that motion of the magnetic fluids is irrotational, it can be described by the potentials $\phi_{1}(x, y, t)$ in the lower fluid and $\phi_{2}(x, y, t)$ in the upper fluid. Then for incompressible fluids, $\phi_{1}(x, y, t)$ and $\phi_{2}(x, y, t)$ satisfy the Laplace equation. By combination of equations (4) and (6) and considering $\mu_{(i)}$ as constant, we find that magnetic scalar potentials satisfy Laplace equations. Thus, equations governing the velocity potential $\phi$ and the magnetic potential $\Psi$ are

$$
\begin{array}{ll}
\nabla^{2} \phi_{1}=\nabla^{2} \Psi_{1}=0 & -h_{1}<y<\eta(x, t) \\
\nabla^{2} \phi_{2}=\nabla^{2} \Psi_{2}=0 & \eta(x, t)<y<h_{2}
\end{array}
$$

\subsubsection{Boundary conditions}

- Boundary conditions at the rigid surfaces $y=-h_{1}$ and $y=h_{2}$ are:

$$
\begin{aligned}
& \phi_{1 y}=0 \\
& \phi_{2 y}=0
\end{aligned}
$$

- Tangential components of the magnetic field vanish on boundaries $y=-h_{1}$ and $y=h_{2}$ are

$$
\begin{aligned}
& \Psi_{1 x}=0 \\
& \Psi_{2 x}=0
\end{aligned}
$$

For small amplitude motion case, nonlinear terms can be ignored in the free interface conditions and taken at the free interface $y=0$.

- Mass conservation across the interface is given as

$$
\rho_{1}\left(\phi_{1 y}-\eta_{t}-v_{1} \eta_{x}\right)=\rho_{2}\left(\phi_{2 y}-\eta_{t}-v_{2} \eta_{x}\right)
$$

- Continuity of magnetic field tangential component at the interface, i.e.

$$
\left(\Psi_{1 x}-\Psi_{2 x}\right)-\eta_{x}\left(H_{02}^{(1)}-H_{02}^{(2)}\right)=0
$$

- Continuity of the magnetic displacement normal component at the interface, i.e.

$$
\eta_{x}\left(\mu_{1} H_{01}^{(1)}-\mu_{2} H_{01}^{(2)}\right)+\left(\mu_{1} \Psi_{1 y}-\mu_{2} \Psi_{2 y}\right)=0
$$

- Energy conservation condition at interface, gives

$$
\rho_{1}\left(\phi_{1 y}-\eta_{t}-v_{1} \eta_{x}\right)=\alpha \eta
$$

where

$$
\begin{aligned}
& \alpha=\frac{G}{L}\left(\frac{1}{h_{1}}+\frac{1}{h_{2}}\right) \\
& G=\frac{K_{2}\left(T_{0}-T_{2}\right)}{h_{2}}=\frac{K_{1}\left(T_{1}-T_{0}\right)}{h_{1}}
\end{aligned}
$$

$G$ is the equilibrium heat flux and $K_{1}$ and $K_{2}$ are the lower and upper thermal conductivities, respectively. $L$ is the latent heat released when the fluid is transformed from phase 1 to phase 2 .

- Dynamic condition at the interface:

$$
\begin{aligned}
& \rho_{1} \phi_{1 t}-\rho_{2} \phi_{2 t}+\left(\rho_{1}-\rho_{2}\right) g \eta+\rho_{1} v_{1} \phi_{1 x}-\rho_{2} v_{2} \phi_{2 x} \\
& +\mu_{1} H_{01}^{(1)} \Psi_{1 x}-\mu_{2} H_{01}^{(2)} \Psi_{2 x}-\mu_{1} H_{02}^{(1)} \Psi_{1 y} \\
& +\mu_{2} H_{02}^{(2)} \Psi_{2 y}-\sigma \eta_{x x}=0
\end{aligned}
$$

\subsubsection{Equations solution}

The solutions of equations (8) and (9) with the conditions (10)-(17), we get

$$
\begin{gathered}
\eta=A e^{i(k x-\omega t)} \\
\phi_{1}=\frac{A}{k}\left[-i \omega+\left(\frac{\alpha}{\rho_{1}}+i k v_{1}\right)\right] \frac{\cosh \left[k\left(y+h_{1}\right)\right]}{\sinh \left(k h_{1}\right)} e^{i(k x-\omega t)}
\end{gathered}
$$




$$
\begin{aligned}
& \phi_{2}=-\frac{A}{k}\left[-i \omega+\left(\frac{\alpha}{\rho_{2}}+i k v_{2}\right)\right] \frac{\cosh \left[k\left(y-h_{2}\right)\right]}{\sinh \left(k h_{2}\right)} e^{i(k x-\omega t)} \\
& \Psi_{1}=A\left[-i \sinh \left(k h_{2}\right)\left(\mu_{2} H_{01}^{(2)}-\mu_{1} H_{01}^{(1)}\right)\right. \\
& \left.-\mu_{2} \cosh \left(k h_{2}\right)\left(H_{02}^{(2)}-H_{02}^{(1)}\right)\right] \frac{\sinh \left[k\left(y+h_{1}\right)\right]}{\beta} \\
& \times e^{i(k x-\omega t)} \\
& \Psi_{2}=A\left[-i \sinh \left(k h_{1}\right)\left(\mu_{2} H_{01}^{(2)}-\mu_{1} H_{01}^{(1)}\right)\right. \\
& \left.+\mu_{1} \cosh \left(k h_{1}\right)\left(H_{02}^{(2)}-H_{02}^{(1)}\right)\right] \frac{\sinh \left[k\left(y-h_{2}\right)\right]}{\beta} \\
& \times e^{i(k x-\omega t)}
\end{aligned}
$$

where

$\beta=\mu_{2} \sinh \left(k h_{1}\right) \cosh \left(k h_{2}\right)+\mu_{1} \cosh \left(k h_{1}\right) \sinh \left(k h_{2}\right)$

\subsubsection{Dispersion relation}

Substituting the values of $\eta, \phi_{1}, \phi_{2}, \Psi_{1}$ and in $\Psi_{2}$ equation (18), we get following dispersion relation:

$$
a_{0} \omega^{2}+\left(-b_{1}+i a_{1}\right) \omega+\left(a_{2}-i b_{2}\right)=0
$$

where

$$
\begin{aligned}
& a_{0}=\rho_{1} \operatorname{coth}\left(k h_{1}\right)+\rho_{2} \operatorname{coth}\left(k h_{2}\right) \\
& a_{1}=\alpha\left(\operatorname{coth}\left(k h_{1}\right)+\operatorname{coth}\left(k h_{2}\right)\right) \\
& a_{2}=k\left[\left(\rho_{2}-\rho_{1}\right) g-\sigma k^{2}\right. \\
& \left.+k\left(\rho_{1} v_{1}^{2} \operatorname{coth}\left(k h_{1}\right)+\rho_{2} v_{2}^{2} \operatorname{coth}\left(k h_{2}\right)\right)\right] \\
& -\frac{k^{2}\left(\mu_{2}-\mu_{1}\right)^{2}}{\beta}\left[H_{01}^{(1)^{2}} \sinh \left(k h_{1}\right) \sinh \left(k h_{2}\right)\right. \\
& \left.-\frac{\mu_{1}}{\mu_{2}} H_{02}^{(1)^{2}} \cosh \left(k h_{1}\right) \cosh \left(k h_{2}\right)\right] \\
& b_{1}=2 k\left(\rho_{1} v_{1} \operatorname{coth}\left(k h_{1}\right)+\rho_{2} v_{2} \operatorname{coth}\left(k h_{2}\right)\right) \\
& b_{2}=\alpha k\left(v_{1} \operatorname{coth}\left(k h_{1}\right)+v_{2} \operatorname{coth}\left(k h_{2}\right)\right)
\end{aligned}
$$

Before dealing with the dispersion relation in detail, we first consider the case where mass and heat transfer effects across the interface is negligible (i.e. $\alpha=0$ ). Which means that dispersion relation is reduced to

$$
a_{0} \omega^{2}-b_{1} \omega+a_{2}=0
$$

It follows that the system is stable, provided that

$$
b_{1}^{2}-4 a_{0} a_{2}>0
$$

or

$$
\begin{aligned}
& \left(\rho_{2}-\rho_{1}\right) g-\sigma k^{2} \\
& +\frac{k \rho_{1} \rho_{2}\left(v_{1}-v_{2}\right)^{2} \operatorname{coth}\left(k h_{1}\right) \operatorname{coth}\left(k h_{2}\right)}{\rho_{1} \operatorname{coth}\left(k h_{1}\right)+\rho_{2} \operatorname{coth}\left(k h_{2}\right)} \\
& -\frac{k^{2}\left(\mu_{2}-\mu_{1}\right)^{2}}{\beta}\left[H_{01}^{(1)^{2}} \sinh \left(k h_{1}\right) \sinh \left(k h_{2}\right)\right. \\
& \left.-\frac{\mu_{1}}{\mu_{2}} H_{02}^{(1)^{2}} \cosh \left(k h_{1}\right) \cosh \left(k h_{2}\right)\right]<0
\end{aligned}
$$

When the parameter $\alpha$ (the coefficient of mass and heat transfer) is not null. We know Applying from the Routh-Hurwitz criterion that necessary and sufficient conditions for stability (in other words, to have the imaginary part of $\omega$ less than zero) are

$$
a_{1}>0
$$

and

$$
a_{0} b_{2}^{2}-a_{1} b_{1} b_{2}+a_{2} a_{1}^{2}<0
$$

or

$$
\begin{aligned}
& \left(\rho_{2}-\rho_{1}\right) g-\sigma k^{2} \\
& +\frac{k \rho_{1} \rho_{2}\left(v_{1}-v_{2}\right)^{2} \operatorname{coth}\left(k h_{1}\right) \operatorname{coth}\left(k h_{2}\right)}{\rho_{1} \operatorname{coth}\left(k h_{1}\right)+\rho_{2} \operatorname{coth}\left(k h_{2}\right)} \\
& -\frac{k^{2}\left(\mu_{2}-\mu_{1}\right)^{2}}{\beta}\left[H_{01}^{(1)^{2}} \sinh \left(k h_{1}\right) \sinh \left(k h_{2}\right)\right. \\
& \left.-\frac{\mu_{1}}{\mu_{2}} H_{02}^{(1)^{2}} \cosh \left(k h_{1}\right) \cosh \left(k h_{2}\right)\right]<0
\end{aligned}
$$

Since $\alpha$ is always positive, condition (28) is automatically satisfied.

\section{Results and discussion}

In what follows, numerical illustrations of the stability criterion are made. The calculations will be performed with equality (27) and (30). This study is based on the effect of an oblique magnetic field as well as mass and heat transfer parameters on the stability conditions, the numerical estimations took into account, in particular, the effect of these parameters.

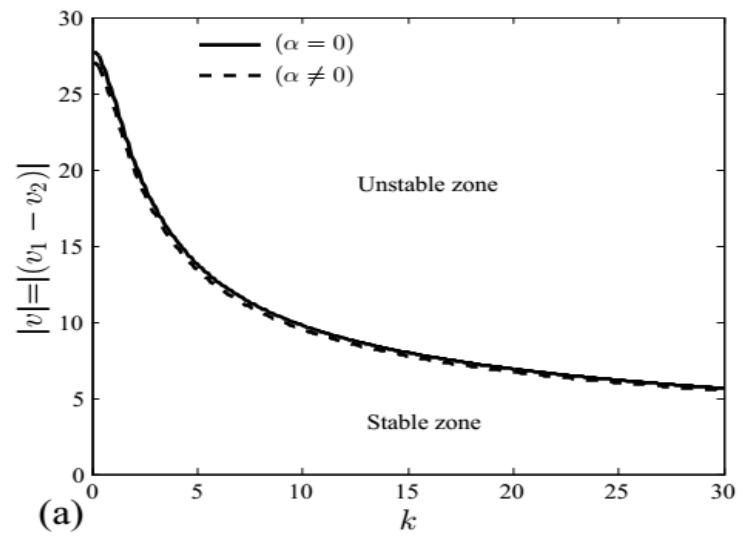



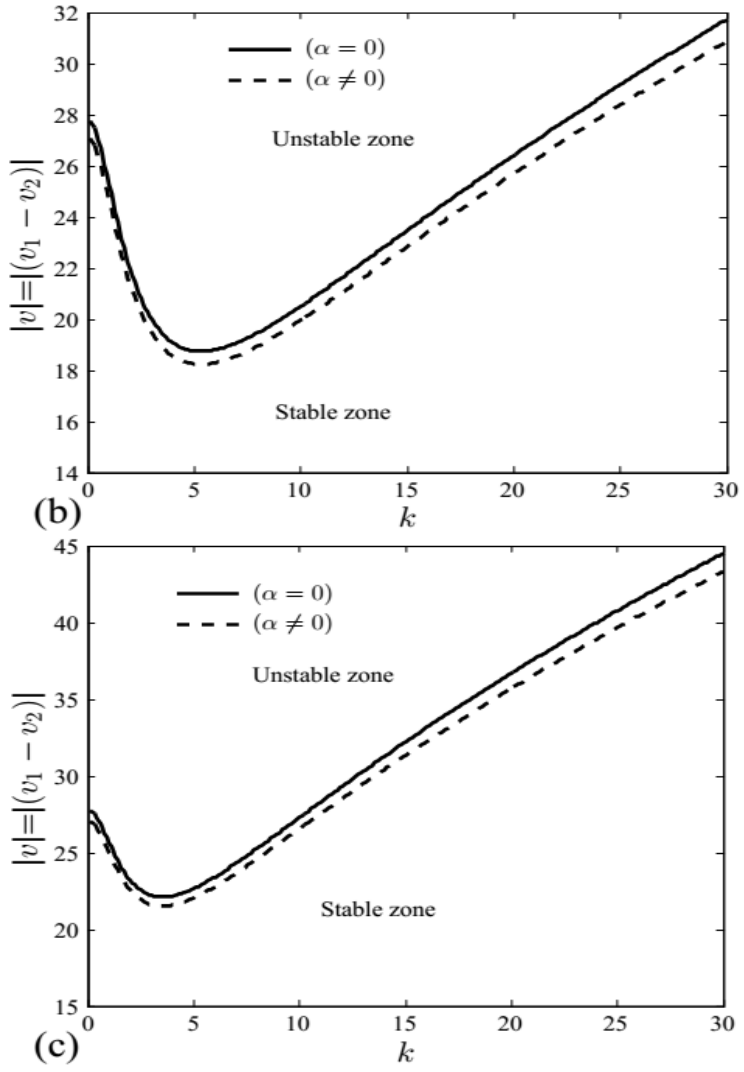

Fig. 1. Stability diagram of velocity versus wave number $k$ in the absence of oblique magnetic field, for $\rho_{1}=0.8 \mathrm{~g} / \mathrm{cm}^{3}, \rho_{2}=0.5 \mathrm{~g} / \mathrm{cm}^{3}, h_{1}=0.5 \mathrm{~cm}, h_{2}=1 \mathrm{~cm}$ and $g=981 \mathrm{~cm} / \mathrm{s}^{2}$. (-) Without of mass and heat transfer. (----) With of mass and heat transfer. (a) $\sigma=0 d y n e / \mathrm{cm}$ (b)

$$
\sigma=10 d y n e / \mathrm{cm} \text { (c) } \sigma=30 d y n e / \mathrm{cm} \text {. }
$$

Figures (1a)-(1c) show examples of a stability diagram of the velocity as a function of the wave number, in the absence of a oblique magnetic field, with and without mass and heat transfer, we obtain the socalled "neutral curve" separates the stable region from the unstable region. It is apparent that the presence of mass and heat transfer gives a new unstable region bounded between these transition curves in the presence and absence of surface tension. We find that the unstable region decreases with the increase of the surface tension.

Figures (2a)-(2b) show the influence of the oblique magnetic field, with and without of mass and heat transfer. A stable and unstable area was recently formed due to the presence of this oblique field. We observe that the unstable region increases as the oblique magnetic field increases. It is therefore concluded that the magnetic field has a destabilizing effect on the system.
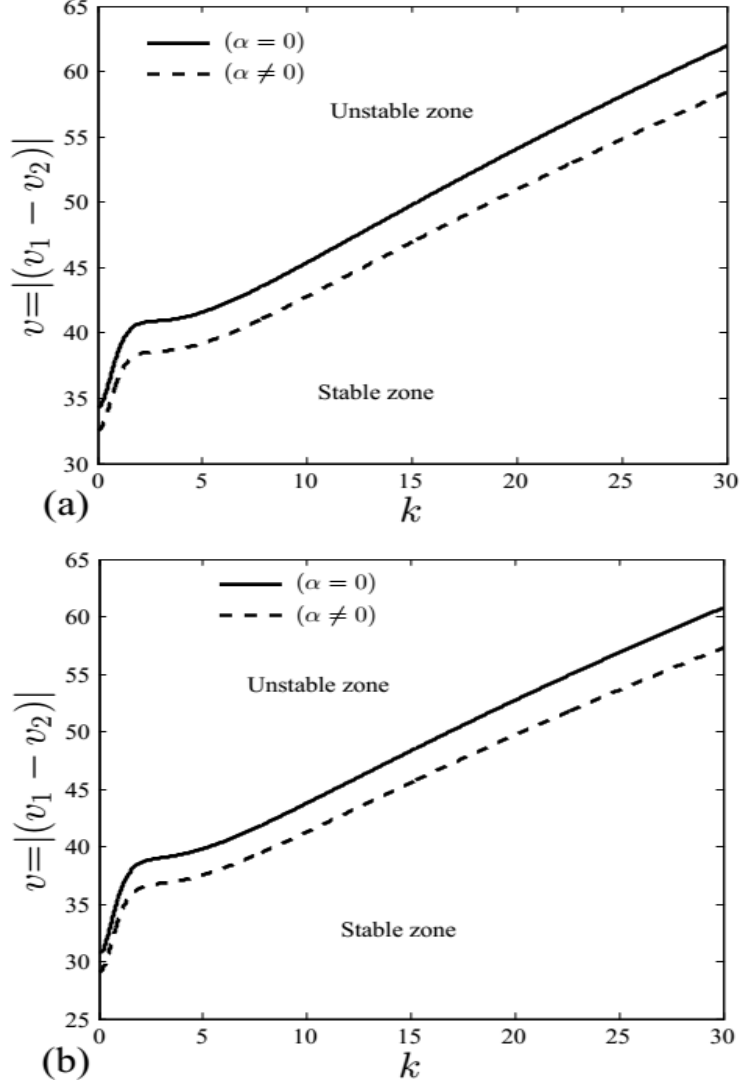

Fig. 2. Neutral curves for velocity versus wave number $k$ in the presence of oblique magnetic field, for

$$
\begin{gathered}
\rho_{1}=0.8 \mathrm{~g} / \mathrm{cm}^{3}, \rho_{2}=0.4 \mathrm{~g} / \mathrm{cm}^{3}, h_{1}=0.5 \mathrm{~cm}, \\
h_{2}=1 \mathrm{~cm}, \mu_{1}=0.01 \mathrm{H} / \mathrm{m}, \mu_{2}=0.7 \mathrm{H} / \mathrm{m} \text {, (a) } \\
H_{01}^{(1)}=20 \mathrm{~A} / \mathrm{m}, H_{02}^{(1)}=30 \mathrm{~A} / \mathrm{m}, \text { (b) } H_{01}^{(1)}=20 \mathrm{~A} / \mathrm{m},
\end{gathered}
$$$$
H_{02}^{(1)}=50 A / m, \sigma=25 d y n e / \mathrm{cm} \text { and } \mathrm{g}=981 \mathrm{~cm} / \mathrm{s}^{2} \text {. }
$$

(-) Without of mass and heat transfer. (-----) With of mass and heat transfer.

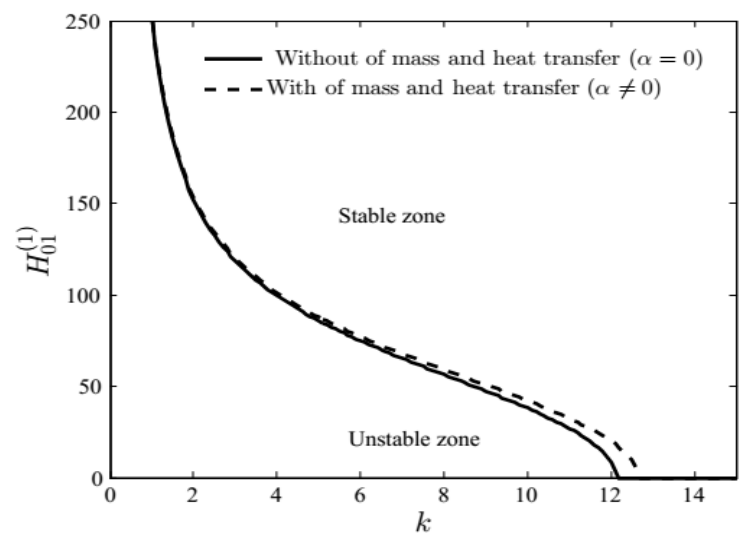

Fig. 3. Stable and unstable zones evolution for the horizontal magnetic field $H_{01}^{(1)}$ versus wave number $k$, for $H_{02}^{(1)}=0$,

$$
\begin{gathered}
\rho_{1}=1.2 \mathrm{~g} / \mathrm{cm}^{3}, \rho_{2}=5.1 \mathrm{~g} / \mathrm{cm}^{3}, h_{1}=0.5 \mathrm{~cm}, \\
h_{2}=1 \mathrm{~cm}, \mu_{1}=0.05 \mathrm{H} / \mathrm{m}, \mu_{2}=0.2 \mathrm{H} / \mathrm{m}, \\
v_{1}=12 \mathrm{~cm} / \mathrm{s}, v_{2}=5 \mathrm{~cm} / \mathrm{s}, \sigma=30 \mathrm{dyne} / \mathrm{cm} \text { and } \\
g=981 \mathrm{~cm} / \mathrm{s}^{2} .
\end{gathered}
$$


Figure 3 shows the evolution of stable and unstable zones for the horizontal magnetic field as a function of the wave number. We note that the destabilizing effect of parameter $\alpha$, appears particularly at large wave number values.

In the stability diagram as given in figure 4, where $H_{02}^{(1)}$ is the vertical magnetic field and $k$ is the wave number, we obtain the so-called neutral curve separates the stable region from the unstable region with and without of mass and heat transfer. It is clear that the vertical field is strictly destabilizing.

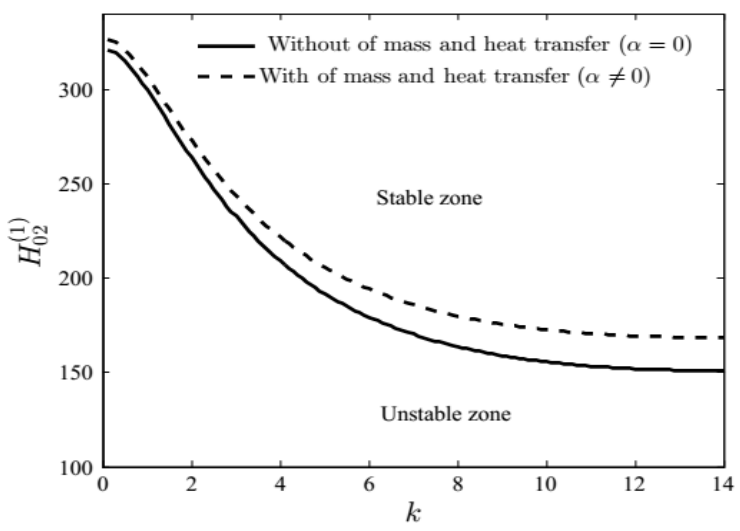

Fig. 4. Stable and unstable zones evolution for the vertical magnetic field $H_{02}^{(1)}$ versus wave number $k$, for $H_{01}^{(1)}=0$,

$$
\begin{gathered}
\rho_{1}=5.1 \mathrm{~g} / \mathrm{cm}^{3}, \rho_{2}=1.2 \mathrm{~g} / \mathrm{cm}^{3}, h_{1}=0.5 \mathrm{~cm}, \\
h_{2}=1 \mathrm{~cm}, \mu_{1}=0.05 \mathrm{H} / \mathrm{m}, \mu_{2}=0.2 \mathrm{H} / \mathrm{m}, \\
v_{1}=12 \mathrm{~cm} / \mathrm{s}, v_{2}=5 \mathrm{~cm} / \mathrm{s}, \sigma=20 d y n e / \mathrm{cm} \text { and } \\
g=981 \mathrm{~cm} / \mathrm{s}^{2} .
\end{gathered}
$$

\section{Conclusion}

The effect of the oblique magnetic field on the linear analysis of the Kelvin-Helmholtz instability has been investigated when there is heat and mass transfer across the magnetic fluids interface. Equations governing linear motion of free interface have been solved using variables separation method. The dispersion relation is obtained which is a quadratic equation, with complex coefficients. We have studied the dispersion relation analytically and numerically in the case of a horizontal vertical and oblique magnetic field. The stability condition is obtained by applying Routh Hurwitz criterion for stability. Mass and heat transfer effects are revealed through one single parameter $\alpha$. It is observed that the heat and mass transfer has a destabilizing effect on the stability of the system and this effect is enhanced in the presence of a magnetic field. For the Kelvin-Helmholtz problem, the stability criterion is independent of mass and heat transfer parameter, but is different from that in the same problem without mass and heat transfer.

\section{References}

1. D.Y. Hsieh, Phys. Fluids 21, 745 (1978)

2. D.S. Lee, J. Phys. A: Math. Gen 38, 2803 (2005)

3. R. Asthana, G.S. Agrawal, Physica A 382, 389 (2007)

4. A.R.F. Elhefnawy, A. Radwan, Physica A 190, 330 (1992)

5. A.R.F. Elhefnawy, Z. Angew. Math. Phys. 77, 19 (1997) 